\title{
Article \\ Genome-Wide Identification and Expression Profile of OSCA Gene Family Members in Triticum aestivum $\mathrm{L}$.
}

\author{
Kai Tong ${ }^{1}{ }^{1}$, Xinyang $\mathrm{Wu}^{2}$, Long $\mathrm{He}^{3}$, Shiyou Qiu ${ }^{1}$, Shuang Liu ${ }^{1}$, Linna Cai ${ }^{1}$, Shaofei Rao ${ }^{1, *}$ \\ and Jianping Chen $1, * \mathbb{B}$
}

1 State Key Laboratory for Managing Biotic and Chemical Threats to the Quality and Safety of Agro-Products, Key Laboratory of Biotechnology in Plant Protection of Ministry of Agriculture and Zhejiang Province, Institute of Plant Virology, Ningbo University, Ningbo 315211, China; 2011074042@nbu.edu.cn (K.T.); qiushiyoudyzx@126.com (S.Q.); liushuang4060@126.com (S.L.); cln7471lcy@163.com (L.C.)

2 College of Life Science, China Jiliang University, Hangzhou 310058, China; xy_wu@zju.edu.cn

3 Ministry of Agriculture Key Lab of Molecular Biology of Crop Pathogens and Insects, Institute of Biotechnology, College of Agriculture \& Biotechnology, Zhejiang University, Hangzhou 310058, China; hnndhelong2@163.com

* Correspondence: raoshaofei@nbu.edu.cn (S.R.); jianpingchen@nbu.edu.cn (J.C.)

Citation: Tong, K.; Wu, X.; He, L.; Qiu, S.; Liu, S.; Cai, L.; Rao, S.; Chen, J. Genome-Wide Identification and Expression Profile of OSCA Gene Family Members in Triticum aestivum L. Int. J. Mol. Sci. 2022, 23, 469. https://doi.org/10.3390/ijms23010469

Academic Editor: Frank M. You

Received: 10 December 2021

Accepted: 30 December 2021

Published: 31 December 2021

Publisher's Note: MDPI stays neutral with regard to jurisdictional claims in published maps and institutional affiliations.

Copyright: (C) 2021 by the authors. Licensee MDPI, Basel, Switzerland. This article is an open access article distributed under the terms and conditions of the Creative Commons Attribution (CC BY) license (https:// creativecommons.org/licenses/by/ $4.0 /)$.

\begin{abstract}
Hyperosmolality and various other stimuli can trigger an increase in cytoplasmic-free calcium concentration $\left(\left[\mathrm{Ca}^{2+}\right]_{\mathrm{cyt}}\right)$. Members of the Arabidopsis thaliana (L.) reduced hyperosmolality-gated calcium-permeable channels (OSCA) gene family are reported to be involved in sensing extracellular changes to trigger hyperosmolality-induced $\left[\mathrm{Ca}^{2+}\right]_{\mathrm{cyt}}$ increases and controlling stomatal closure during immune signaling. Wheat (Triticum aestivum L.) is a very important food crop, but there are few studies of its OSCA gene family members. In this study, 42 OSCA members were identified in the wheat genome, and phylogenetic analysis can divide them into four clades. The members of each clade have similar gene structures, conserved motifs, and domains. TaOSCA genes were predicted to be regulated by cis-acting elements such as STRE, MBS, DRE1, ABRE, etc. Quantitative PCR results showed that they have different expression patterns in different tissues. The expression profiles of 15 selected TaOSCAs were examined after PEG (polyethylene glycol), NaCl, and ABA (abscisic acid) treatment. All 15 TaOSCA members responded to PEG treatment, while TaOSCA12/-39 responded simultaneously to PEG and ABA. This study informs research into the biological function and evolution of TaOSCA and lays the foundation for the breeding and genetic improvement of wheat.
\end{abstract}

Keywords: abiotic stress; reduced hyperosmolality-gated calcium-permeable channels; wheat; whole genome expression analysis

\section{Introduction}

Under natural conditions, plants encounter a variety of biotic and abiotic stresses [1,2]. Plants resist these stresses by sensing and transmitting signals in a variety of ways that regulate responses and gene expression, thereby producing appropriate physiological and morphological changes [3-5]. In the process of signal perception, calcium ions $\left(\mathrm{Ca}^{2+}\right)$ are important second messengers when plants respond to stress [6]. Under osmotic stress, plants induce a rapid increase in intracellular free $\mathrm{Ca}^{2+}$ concentration $\left(\left[\mathrm{Ca}^{2+}\right]_{\text {cyt }}\right)$, thereby inducing the expression of many stress-related genes and regulating the tolerance of plants to osmotic stress $[4,7]$. Blocking hyperosmolality-induced $\left[\mathrm{Ca}^{2+}\right]_{\text {cyt }}$ increase (OICI) interferes with gene expression induced by drought, indicating that the precise regulation of OICI is essential for the activation of many signal transduction pathways triggered by external stimuli [7]. The increase in intracellular $\mathrm{Ca}^{2+}$ concentration is mainly regulated by calcium transport systems such as calcium channels and calcium pumps [8]. Previous studies have shown that osmotic/mechanical stimulus-gated calcium permeable channels 
play the role of osmosensors in bacteria and animals $[9,10]$, which indicates that there may be specific calcium-permeable channels in plants that function as osmosensors [11,12].

Some protein families, including glutamate receptor-like proteins (GLR), cyclic nucleotide gated channels (CNGCs), and annexins, are involved in calcium flux in plant cells [13]. However, none of them can directly respond to pressure signals [14]. In 2014, Yuan et al. used a genetic screening strategy based on calcium imaging to isolate Arabidopsis thaliana (L.) mutants with a low intracellular free calcium concentration under high osmotic stress and characterized the gene reduced hyperosmolality-induced $\left[\mathrm{Ca}^{2+}\right]_{c y t}$ increase 1 (osca1.1) as the previously unknown hyperosmolality-gated calcium-permeable channel [15]. An osca1.1 mutant had impaired osmotic calcium signal transmission in guard cells and root cells and impaired regulation of water transpiration and root growth in response to osmotic stress, indicating that osca1.1 may be an osmosensor in A. thaliana [15]. OSCA1.1 belongs to a 15-member gene family in $A$. thaliana, and homologous genes are found in other plant species and eukaryotes [16-18]. Studies on functional domains indicate that the OSCA gene family encodes a calcium-dependent channel domain (DUF221) [14]. DUF221 belongs to the anoctamin-like family and is homologous to the domain in the anoctamin/TMEM16 channel, which is a Calcium-activated Chloride Channel (CaCC) component and transmembrane channel-like protein (TMC) [19]. DUF221 proteins are a family of osmosensitive calcium-permeable cation channels, which are conserved in eukaryotes [20]. By heterologous expression of $A$. thaliana genes in Chinese Hamster Ovary $(\mathrm{CHO})$ cells loaded with calcium-sensitive dye Fura-2, Hou et al. screened OSCA1.2 as an ion channel that can be activated by hyperosmotic shock. Detailed analysis showed that OSCA1.2 has good permeability to cations such as $\mathrm{Ca}^{2+}, \mathrm{K}^{+}$, and $\mathrm{Na}^{+}$[14]. OSCA3.1 was previously reported to be an early response protein to dehydration stress in A. thaliana [21]. However, Yuan et al. reported that OSCA3.1 knockout mutants displayed normal OICIs, suggesting that the function of OSCA3.1 may be different from that of OSCA1.1 [15].

The perception of biotic and abiotic stresses often leads to the closure of plant stomata $[1,22]$, and the rapid influx of calcium through the plasma membrane plays an important role in this response $[23,24]$. However, the identity of the calcium channel was unclear until Thor et al. recently reported that the A. thaliana $\mathrm{Ca}^{2+}$-permeable channel OSCA1.3 controls stomatal closure in the process of immune signal transduction [25]. OSCA1.3 is permeable to $\mathrm{Ca}^{2+}$ and can be rapidly phosphorylated by the receptor-like cytoplasmic kinase protein BIK1 when sensing Pathogen-Associated Molecular Patterns (PAMPs). Genetic and electrophysiological data reveal that in the process of immune signal transduction, BIK1-mediated $\mathrm{N}$-terminal phosphorylation of OSCA1.3 increases the activity of this channel [25]. However, OSCA1.3 does not regulate stomatal closure induced by abscisic acid (ABA, a plant hormone related to abiotic stress) [25]. In summary, the members of the OSCA family are both conserved and differentiated in function.

OSCA family members thus play a vital role in plant resistance to high osmotic stress and other stimuli. The OSCA gene family has been systematically identified and analyzed in A. thaliana [14,15], rice [16], maize [18], and Vigna radiate L. [17] but not so far in wheat (Triticum aestivum L.), globally one of the most widely grown crops [26]. To explore whether the wheat OSCA gene family has a function in the process of regulating abiotic stress response, we conducted a genome-wide identification of T. aestivum OSCA family members and analyzed their phylogenetic relationships and expression profiles under different tissues and different abiotic stresses in this study. These results lay the foundation for studying the function of the wheat OSCA genes and increase our understanding of the role of plant OSCA genes in general. 


\section{Results}

2.1. Genome-Wide Identification and Naming of TaOSCA Members

We identified the possible OSCA members in Triticum aestivum based on the criterion that the OSCA gene contains a conserved DUF221 functional domain (pfam accession number: 02714). The amino acid sequences of 15 identified $A$. thaliana OSCA members were downloaded from TAIR, and the T. aestivum genome sequence and gene structure annotation files were downloaded from the Ensemble Plants database (http://plants. ensembl.org/Triticum_aestivum/Info/Index, accessed on 29 December 2021). A total of 42 TaOSCAs were identified after two rounds of BLASTP. These members are named TaOSCA1 to TaOSCA42 according to their position on the chromosomes (Table 1 and Supplementary Table S1). The proteins range in size from 469 to 804 amino acids (aa), with most having about 700 aa. Their molecular weights are $53.7-93.4 \mathrm{kDa}$, and the isoelectric points are in the range of 6.59-9.77.

Table 1. Detailed information of the 42 predicted OSCA proteins in T. aestivum.

\begin{tabular}{|c|c|c|c|c|c|c|}
\hline $\begin{array}{c}\text { Gene } \\
\text { Symbol }\end{array}$ & Gene Locus & Chromosome Position & $\begin{array}{l}\text { CDS } \\
(\mathrm{bp})\end{array}$ & $\begin{array}{c}\text { Protein } \\
\text { Length (aa) }\end{array}$ & Theoretical PI & $\begin{array}{c}\text { Molecular } \\
\text { Weight (kDa) }\end{array}$ \\
\hline TaOSCA1 & TraesCS1A02G029300.1 & 1A:13762566:13768572 & 2295 & 764 & 8.94 & 87.7 \\
\hline TaOSCA2 & TraesCS1A02G034100.1 & 1A:16913591:16919361 & 2301 & 766 & 9.06 & 87.9 \\
\hline TaOSCA3 & TraesCS1A02G196800.1 & $1 \mathrm{~A}: 354950050: 354955890$ & 2412 & 803 & 9.15 & 93.4 \\
\hline TaOSCA4 & TraesCS1A02G247300.1 & $1 \mathrm{~A}: 438692887: 438699043$ & 2304 & 767 & 7.33 & 87.6 \\
\hline TaOSCA5 & TraesCS1B02G036500.1 & 1B:17379054:17386839 & 2295 & 764 & 8.81 & 87.8 \\
\hline TaOSCA6 & TraesCS1B02G211400.1 & 1B:384496418:384502005 & 2406 & 801 & 9.12 & 93.1 \\
\hline TaOSCA7 & TraesCS1B02G257900.1 & 1B:453873827:453880440 & 2304 & 767 & 7.73 & 87.6 \\
\hline TaOSCA8 & TraesCS1D02G029500.1 & 1D:11616634:11624592 & 2295 & 764 & 8.87 & 87.8 \\
\hline TaOSCA9 & TraesCS1D02G035600.1 & 1D:16402928:16408856 & 2301 & 766 & 9.09 & 87.9 \\
\hline TaOSCA10 & TraesCS1D02G200300.1 & 1D:282959933:282965842 & 2409 & 802 & 9.16 & 93.2 \\
\hline TaOSCA11 & TraesCS1D02G246500.1 & 1D:339106416:339112794 & 2301 & 766 & 7.73 & 87.5 \\
\hline TaOSCA12 & TraesCS2A02G252100.1 & $2 \mathrm{~A}: 383596822: 383601415$ & 2097 & 698 & 9.17 & 78.1 \\
\hline TaOSCA13 & TraesCS2A02G262100.1 & $2 \mathrm{~A}: 413328225: 413332576$ & 2259 & 752 & 8.83 & 85.1 \\
\hline TaOSCA14 & TraesCS2B02G271900.1 & 2B:373476491:373481152 & 2178 & 725 & 9.29 & 81.2 \\
\hline TaOSCA15 & TraesCS2B02G280100.1 & 2B:386306102:386311122 & 2307 & 768 & 6.59 & 87.4 \\
\hline TaOSCA16 & TraesCS2D02G252800.1 & 2D:304963347:304967946 & 2016 & 671 & 9.27 & 75.4 \\
\hline TaOSCA17 & TraesCS2D02G261900.1 & 2D:318328838:318333878 & 2307 & 768 & 6.72 & 87.4 \\
\hline TaOSCA18 & TraesCS3A02G019900.1 & 3A:11891585:11895285 & 2178 & 725 & 9.42 & 81.7 \\
\hline TaOSCA19 & TraesCS3B02G231400.1 & 3B:349934463:349949455 & 2214 & 737 & 8.58 & 84.3 \\
\hline TaOSCA20 & TraesCS3B02G541500.1 & 3B:779622377:779627065 & 2130 & 709 & 8.97 & 80.0 \\
\hline TaOSCA21 & TraesCS3D02G026700.1 & 3D:9094139:9098190 & 1947 & 648 & 9.51 & 72.5 \\
\hline TaOSCA22 & TraesCS3D02G201300.1 & 3D:238617165:238630499 & 2214 & 737 & 8.58 & 84.2 \\
\hline TaOSCA23 & TraesCS3D02G485000.1 & 3D:581939165:581943412 & 2130 & 709 & 9.01 & 80.1 \\
\hline TaOSCA24 & TraesCS4A02G257600.1 & $4 \mathrm{~A}: 570460851: 570465209$ & 2238 & 745 & 8.89 & 85.7 \\
\hline TaOSCA25 & TraesCS4A02G291700.1 & $4 A: 594680010: 594686528$ & 2301 & 766 & 9.33 & 87.0 \\
\hline TaOSCA26 & TraesCS4B02G022300.1 & 4B:16056589:16063157 & 2301 & 766 & 9.44 & 86.8 \\
\hline TaOSCA27 & TraesCS4B02G023400.1 & $4 \mathrm{~B}: 17037475: 17042314$ & 1410 & 469 & 9.77 & 53.7 \\
\hline TaOSCA28 & TraesCS4B02G056900.1 & $4 \mathrm{~B}: 46623552: 46628077$ & 2238 & 745 & 8.84 & 85.7 \\
\hline TaOSCA29 & TraesCS4B02G335800.1 & 4B:627519072:627524699 & 2385 & 794 & 7.2 & 89.6 \\
\hline TaOSCA30 & TraesCS4D02G020100.1 & 4D:8653451:8660027 & 2229 & 742 & 9.13 & 84.3 \\
\hline TaOSCA31 & TraesCS4D02G057200.1 & 4D:32238120:32242237 & 2238 & 745 & 8.84 & 85.7 \\
\hline TaOSCA32 & TraesCS4D02G331300.1 & 4D:489186164:489190977 & 2385 & 794 & 7.55 & 89.5 \\
\hline TaOSCA33 & TraesCS5A02G012500.1 & 5A:8233156:8239545 & 2415 & 804 & 9.07 & 90.7 \\
\hline TaOSCA34 & TraesCS5A02G012600.1 & $5 \mathrm{~A}: 8240521: 8246498$ & 2043 & 680 & 8.04 & 77.2 \\
\hline TaOSCA35 & TraesCS5A02G071400.1 & 5A:80159622:80167881 & 2097 & 698 & 8.71 & 78.9 \\
\hline TaOSCA36 & TraesCS5A02G505500.1 & $5 A: 670818817: 670823201$ & 2385 & 794 & 7.55 & 89.6 \\
\hline TaOSCA37 & TraesCS5B02G010700.1 & 5B:10438285:10442125 & 2286 & 761 & 8.96 & 86.3 \\
\hline TaOSCA38 & TraesCS5B02G010800.1 & 5B:10442174:10450187 & 2319 & 772 & 9.07 & 87.4 \\
\hline TaOSCA39 & TraesCS5B02G077400.1 & 5B:93218161:93227257 & 2094 & 697 & 8.62 & 79.1 \\
\hline TaOSCA40 & TraesCS5D02G018000.1 & 5D:10520893:10527152 & 2334 & 777 & 9.08 & 88.1 \\
\hline TaOSCA41 & TraesCS5D02G018100.1 & 5D:10537542:10542334 & 2316 & 771 & 8.61 & 87.4 \\
\hline TaOSCA42 & TraesCS5D02G083600.1 & 5D:87182360:87193201 & 2097 & 698 & 8.79 & 79.0 \\
\hline
\end{tabular}




\subsection{Phylogenetic Analysis}

In order to better understand the evolutionary relationship of OSCA family genes in $A$. thaliana and T. aestivum, the amino acid sequences of $15 \mathrm{~A}$. thaliana and $42 \mathrm{~T}$. aestivum members were used to construct a phylogenetic tree. The genes clearly divide into four clades (I to IV; Figure 1). There are 16 TaOSCA members in clade I (TaOSCA1, -2, -5, -8, -9, $-19,-22,-4,-7,-11,-13,-15,-17,-3,-6,-10)$ and seven of $A$. thaliana (AtOSCA1.1-AtOSCA1.7). Clade II contains 18 wheat (TaOSCA20, -23, -39, -42, -35, -24, -31, -28, -25, -26, -27, -30, -33, $-40,-38,-34,-37,-41$ ) and four $A$. thaliana (AtOSCA2.1-AtOSCA 2.4) members. There are five wheat members in clade III (TaOSCA18, -21, -14,-12, -16), together with AtOSCA3.1, and three wheat members in clade IV (TaOSCA36, -29, -32) with AtOSCA4.1 (Figure 1).

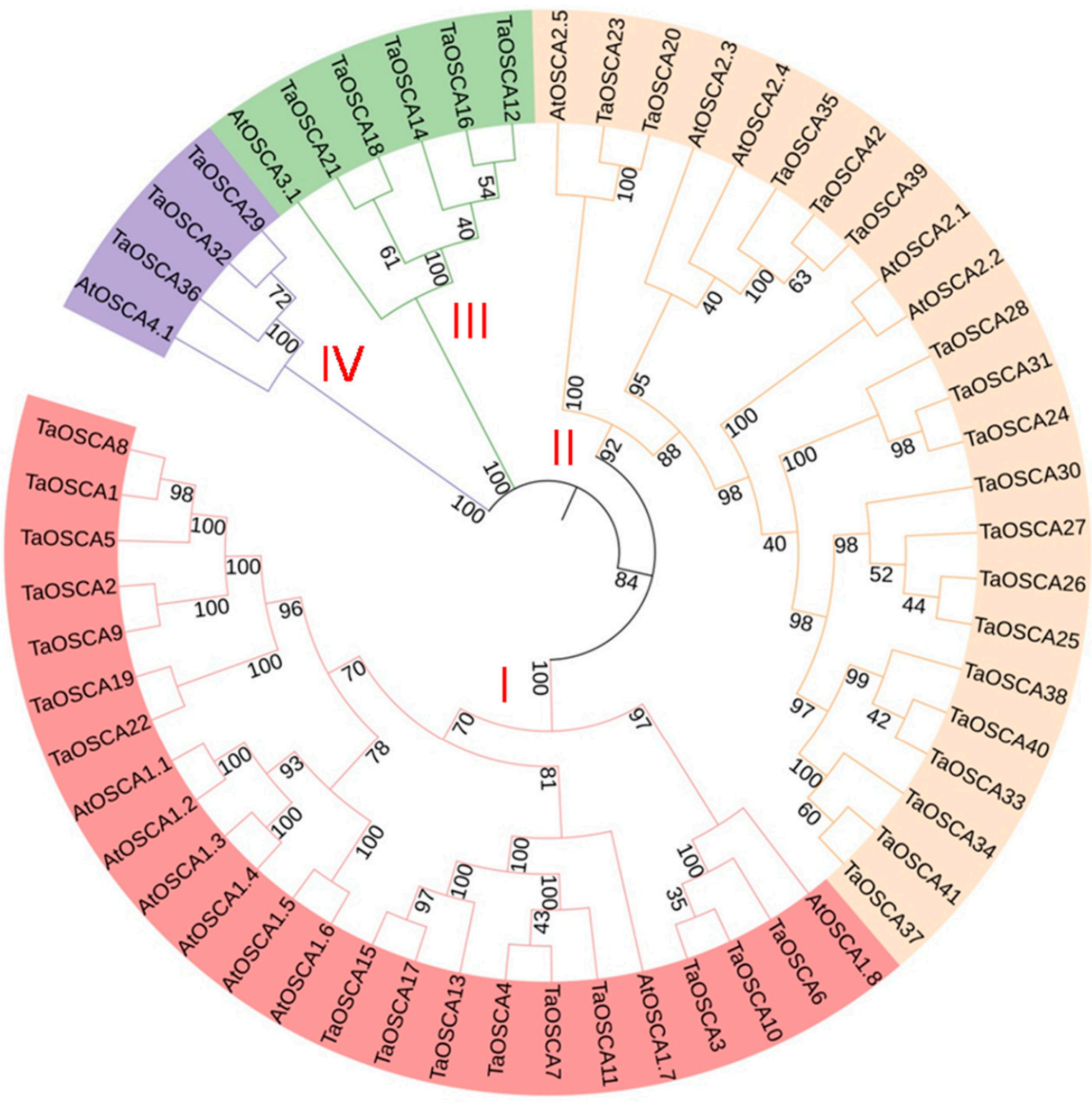

Figure 1. Phylogenetic tree of the OSCA proteins of Triticum aestivum and A. thaliana. The phylogenetic tree was constructed using OSCA amino acid sequences by the neighbor-joining method in MEGA $X$ with 1000 bootstrap replicates. The phylogenetic tree is divided into four groups, which are shown in different colors, and identified by red Roman numerals. 


\subsection{Analysis of TaOSCA Conserved Motifs, Gene Structure, and Domains}

To further understand the evolution of TaOSCA members, we compared the conserved motifs, exon-intron composition, and functional domains of the 42 TaOSCA members. An online MEME analysis predicted a total of 10 conserved motifs (Supplementary Table S2). The vast majority of members contain all 10 predicted motifs, but the three members of clade IV contain only four motifs (Figure 2A,B). TaOSCA12 contains six motifs, TaOSCA27 contains seven motifs, TaOSCA21 contains eight motifs, and TaOSCA19/-22/-16 contain nine motifs (Figure $2 \mathrm{~A}, \mathrm{~B}$ ). The TaOSCA gene structure is relatively conserved with most having about 10 introns, while the three members of the fourth clade have only $2-3$ introns (Figure 2C).

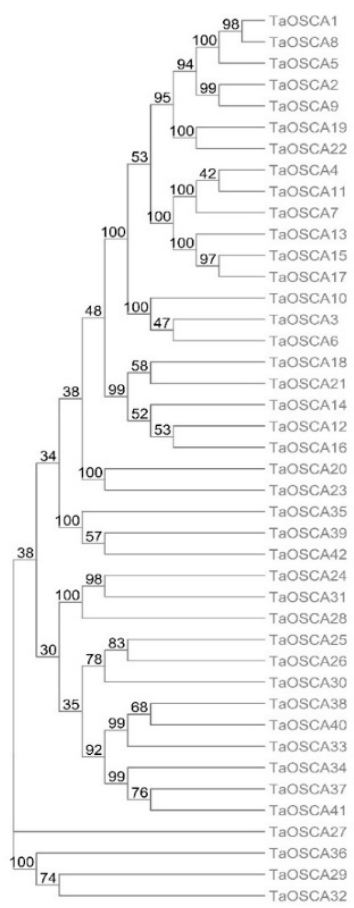

(A)

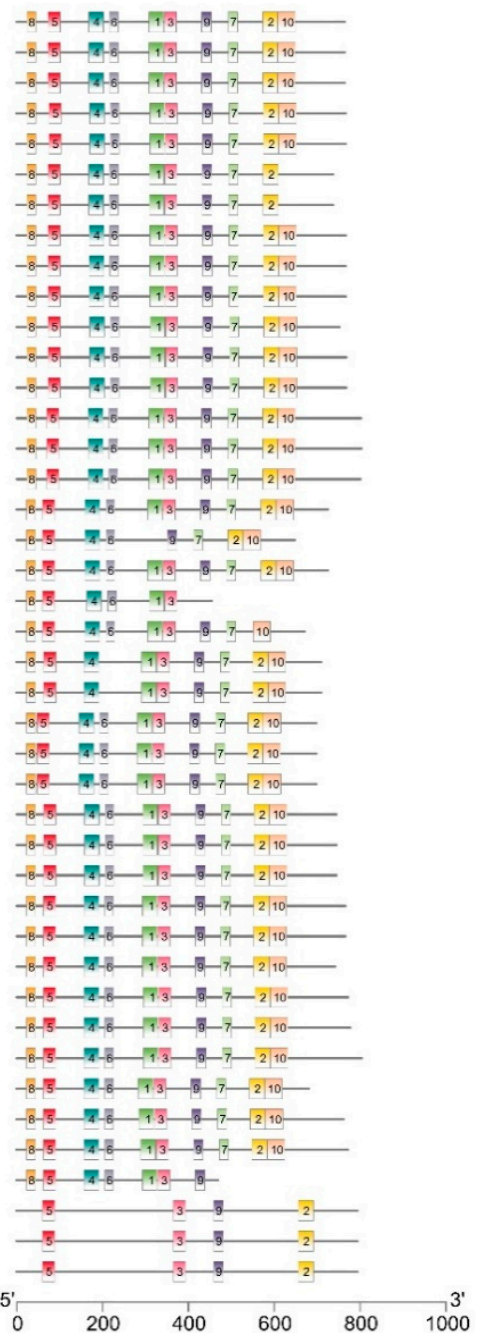

(B)

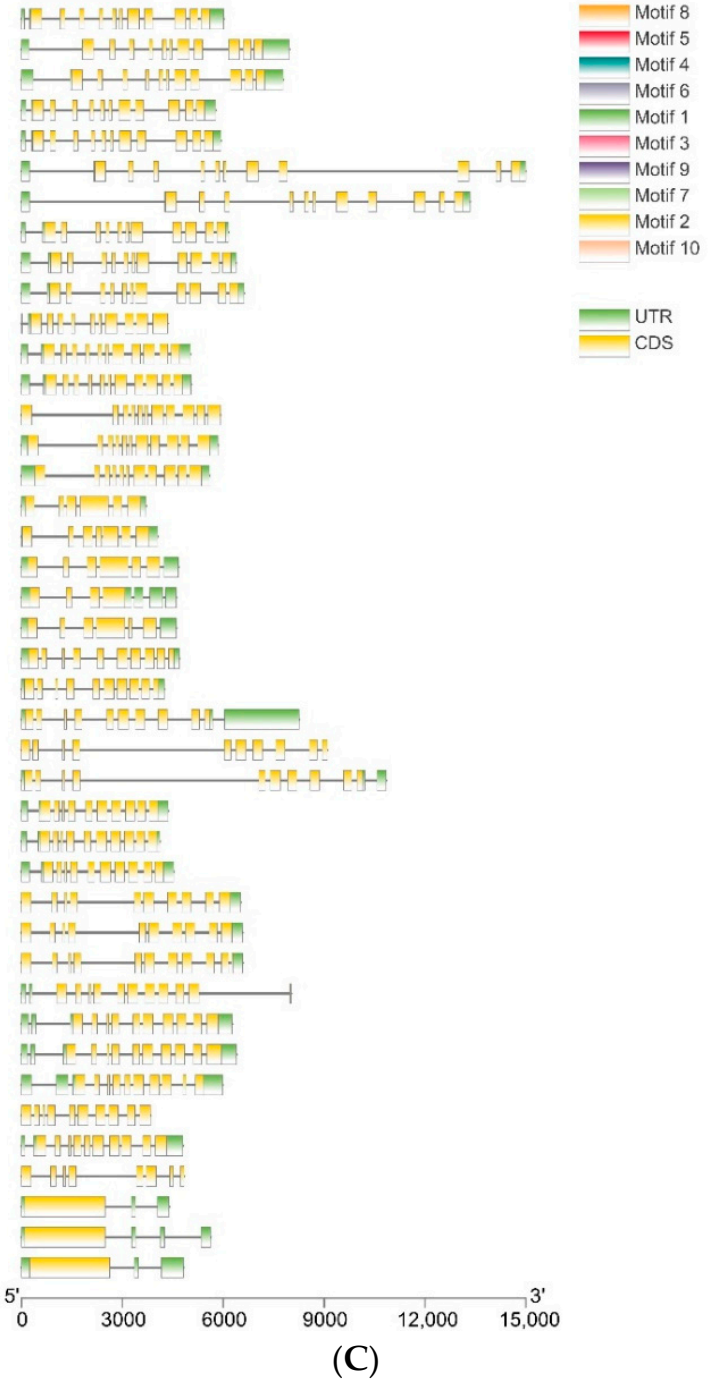

(C)

Figure 2. Analysis of conserved motifs and gene structure of T. aestivum OSCA genes. (A) Phylogenetic tree constructed using the TaOSCA protein sequences. (B) Ten types of conserved motifs predicted in the TaOSCA protein sequences. The different motifs are shown in different color boxes. The sequence information for each motif is provided in Supplementary Table S2. (C) The gene structure of TaOSCA members. Untranslated regions, exons, and introns are shown as green boxes, yellow boxes, and horizontal lines, respectively. 
All 42 members contain the conserved pfam02714 and pfam14703 functional domains. Except for the three members of the fourth clade, the other members also contain a pfam13967 functional domain (Figure 3). Pfam02714 is predicted to be the transmembrane region of the osmosensitive calcium-permeable cation channel $[14,27,28]$. The functional domain of pfam13967 is predicted to be the first three transmembrane regions of transmembrane proteins, which is related to vesicle transport [27]. pfam14703 is predicted to be the cytoplasmic region of an integral membrane protein. This functional domain usually appears before pfam02714 and after pfam13967 [28]. In addition, most TaOSCA genes contain 8-10 transmembrane regions. The exceptions are TaOSCA25/-26/-30/-37, which have 11 transmembrane regions, and TaOSCA12/-27, which have only five (Figure 3).
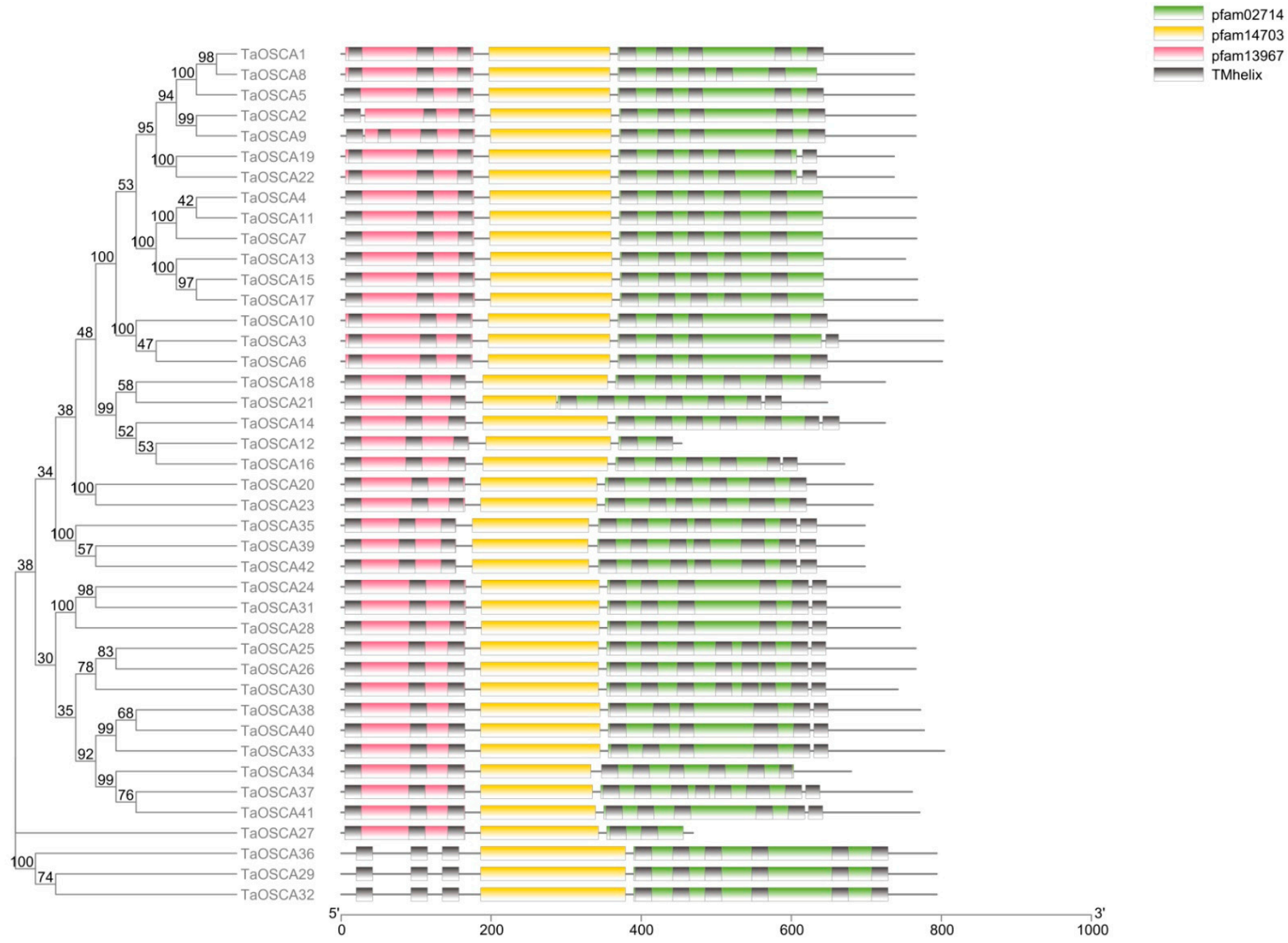

igure 3. Analysis of conserved domains in TaOSCA proteins. Pink, yellow, green, and gray boxes represent the pfam13967, pfam14703, pfam02714, and transmembrane helix domain, respectively. 


\subsection{Prediction of Cis-Acting Elements of the TaOSCA Promoter}

The cis-acting elements in the TaOSCA promoter region were then analyzed, and a total of 107 types of 6542 cis-acting elements were predicted (Supplementary Table S3). These cis-acting elements are related to environmental stress, hormonal response, development, and light response (Figure 4A). A total of 385 elements related to environmental stress are predicted in 10 categories, among which the number of STRE, ARE, and WUN-motif elements is relatively large (Figure 4B). A total of 806 hormone-related components are predicted in 13 categories, of which ABRE, MYC, and CGTCA-motif components are relatively large, mainly related to ABA and JA (Figure $4 \mathrm{C}$ ). This indicates that TaOSCA family genes may be involved in a variety of stress and plant hormone response processes and can effectively promote plant growth and stress resistance.
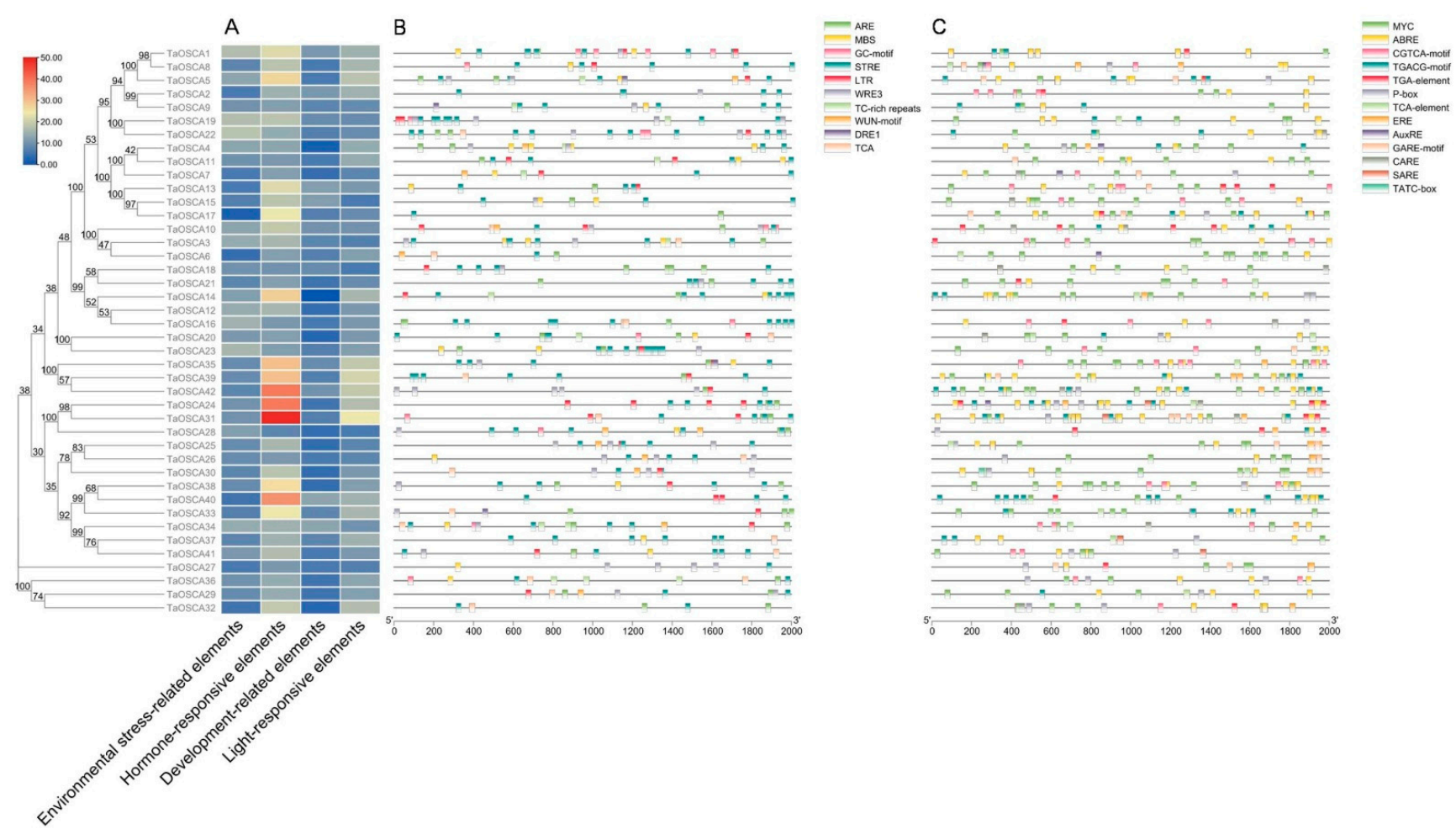

Figure 4. Prediction of cis-acting elements in the TaOSCA promoter regions. (A) Schematic representation of the numbers of four types of cis-acting elements predicted in the promoter region of each TaOSCA member. (B,C) The type, quantity, and position of environmental stress-related elements (B) and hormone-response elements $(\mathbf{C})$ in the promoter region.

\subsection{Expression Patterns of TaOSCA Genes in Different Tissues}

To study the expression pattern of $\mathrm{TaOSCA}$ genes, the expression levels of $15 \mathrm{TaOSCA}$ members representing the different clades were analyzed in five different tissues (root, stem, bottom leaf, middle leaf, top leaf). Gene expression in the roots was always very low or undetectable. TaOSCA37 was expressed most highly in the top leaf, whereas TaOSCA6/-39 were expressed least in this leaf (Figure 5). TaOSCA1/-2/-6/-12/-32/-33/-34/-39/-40 were expressed most highly in the middle leaf (Figure 5). TaOSCA39 was expressed more highly in the bottom leaf than the top leaf, $\mathrm{TaOSCA21/-27/-40} \mathrm{had} \mathrm{lower} \mathrm{expression} \mathrm{levels} \mathrm{in} \mathrm{the}$ bottom leaf, and other members were expressed at similar amounts in the bottom and top leaves (Figure 5). In addition, TaOSCA6/-19/-27/-39 were expressed most highly in the stem (Figure 5). 
TaOSCA1
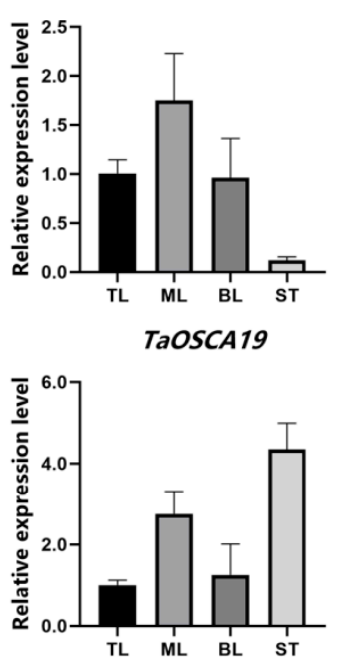

TaOSCA34

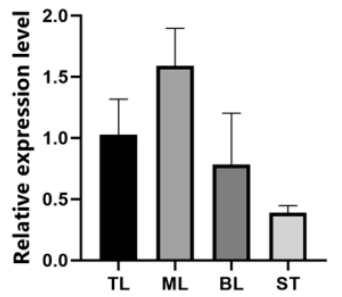

TaOSCA2
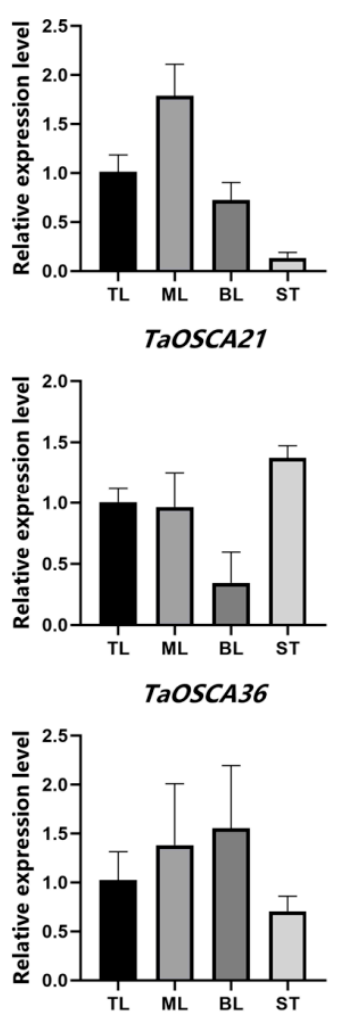

TaOSCA6
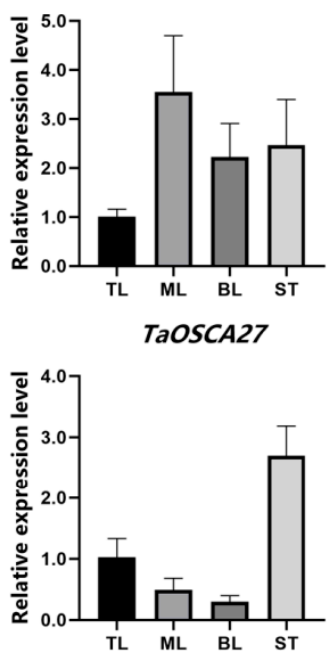

TaOSCA37

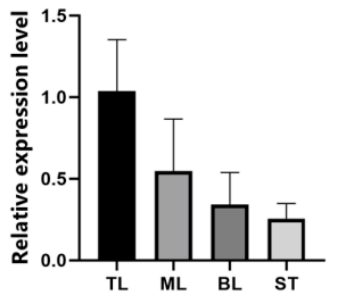

TaOSCA12
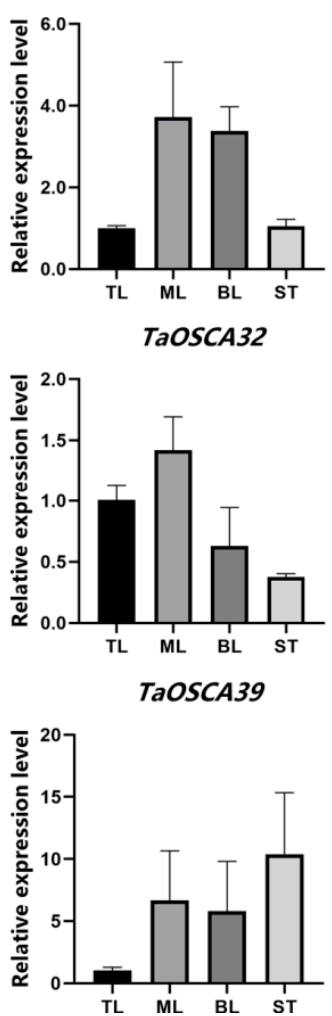

TaOSCA15
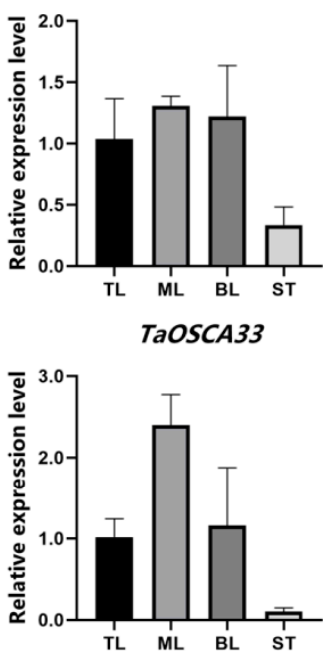

TaOSCA4O

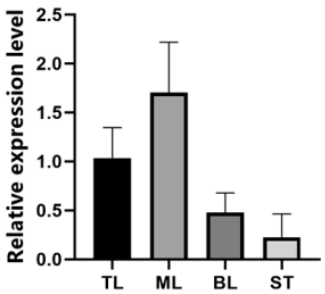

Figure 5. Expression levels of TaOSCAs in different tissues. The mean expression values were calculated from three independent biological replicates relative to that in top leaves. TL: top leaf; MF: middle leaf; BF: bottom leaf; ST: stem.

\subsection{Expression Profiles of TaOSCA Genes in Response to Abiotic Stress Treatments}

PEG (polyethylene glycol) and $\mathrm{NaCl}$ stress can cause similar cell damage, leading to osmotic stress [29]. Plants adapt to and respond to drought and salt stress by inducing the expression of a series of genes. ABA (abscisic acid) is an important plant hormone that regulates the expression of stress response genes in plants [30]. In order to explore the response of the TaOSCA genes to these three abiotic stresses, we analyzed the expression profiles of 15 representative members treated with exogenous PEG, $\mathrm{NaCl}$, or ABA for $4 \mathrm{~h}$, $12 \mathrm{~h}$, and $24 \mathrm{~h}$, respectively. All 15 test genes were all up-regulated at 12 and $24 \mathrm{~h}$ after PEG treatment, but some members were significantly down-regulated at $4 \mathrm{~h}$ (Figure 6). $\mathrm{TaOSCA} 21 /-39$ showed the largest increases at $24 \mathrm{~h}$, up 12 times and 15 times, respectively (Figure 6).

Following $\mathrm{NaCl}$ treatment, most of the members were down-regulated at $4 \mathrm{~h}$ but with no effect at 12 and $24 \mathrm{~h}$, including TaOSCA2/-6/-12/-15/-19//-21/-27/-32/-33/-34/-36/$37 /-40$ (Figure 6). TaOSCA39 was down-regulated at $4 \mathrm{~h}$, unchanged at $12 \mathrm{~h}$, and doubled at $24 \mathrm{~h}$. TaOSCA1 was significantly inhibited at 4 and $12 \mathrm{~h}$, but unchanged at $24 \mathrm{~h}$ (Figure 6).

Following ABA treatment, TaOSCA21 was up-regulated by five times at $24 \mathrm{~h}, \mathrm{TaOSCA} 39$ was up-regulated by five times at $12 \mathrm{~h}$ and $24 \mathrm{~h}$, and TaOSCA40 was up-regulated by two times at $12 \mathrm{~h}$. Except for TaOSCA21/-39, the other members were all down-regulated at $4 \mathrm{~h}$ (Figure 6). 
TaOSCA1
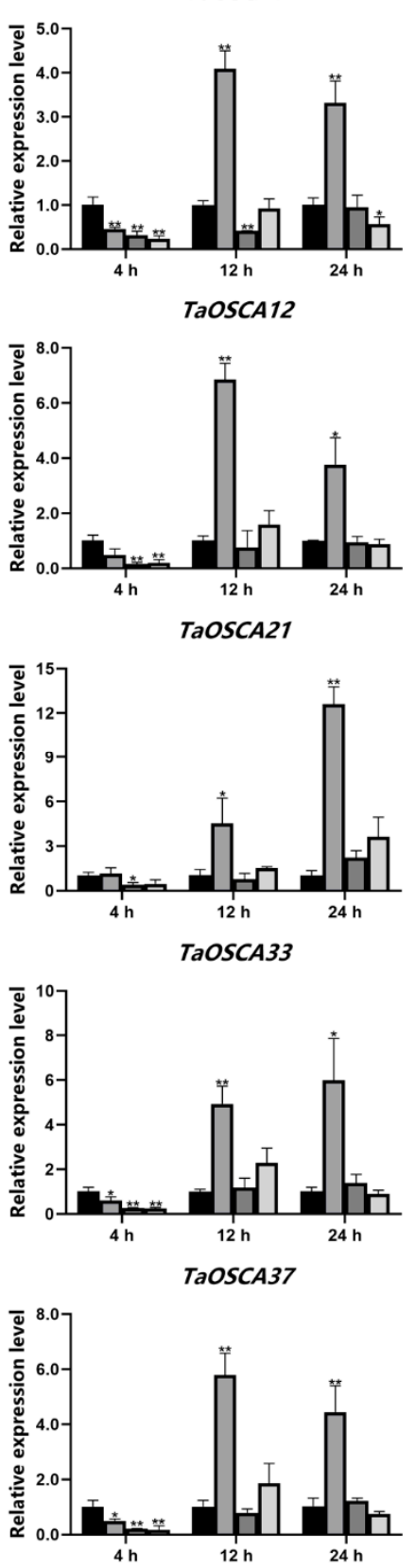

Mock 口 PEG 口 Nacl

TaOSCA2
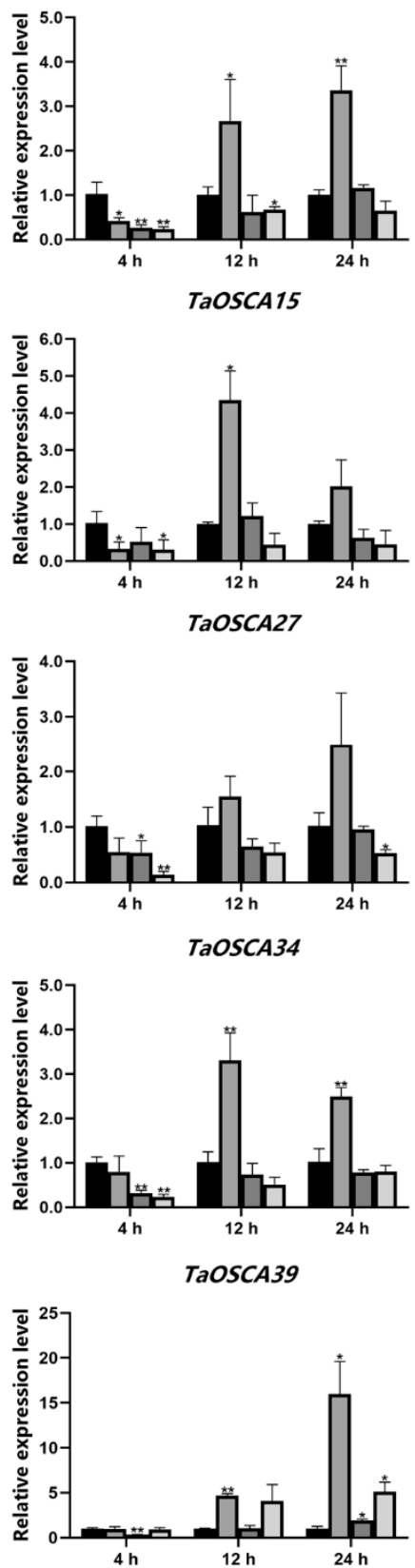

$A B A$
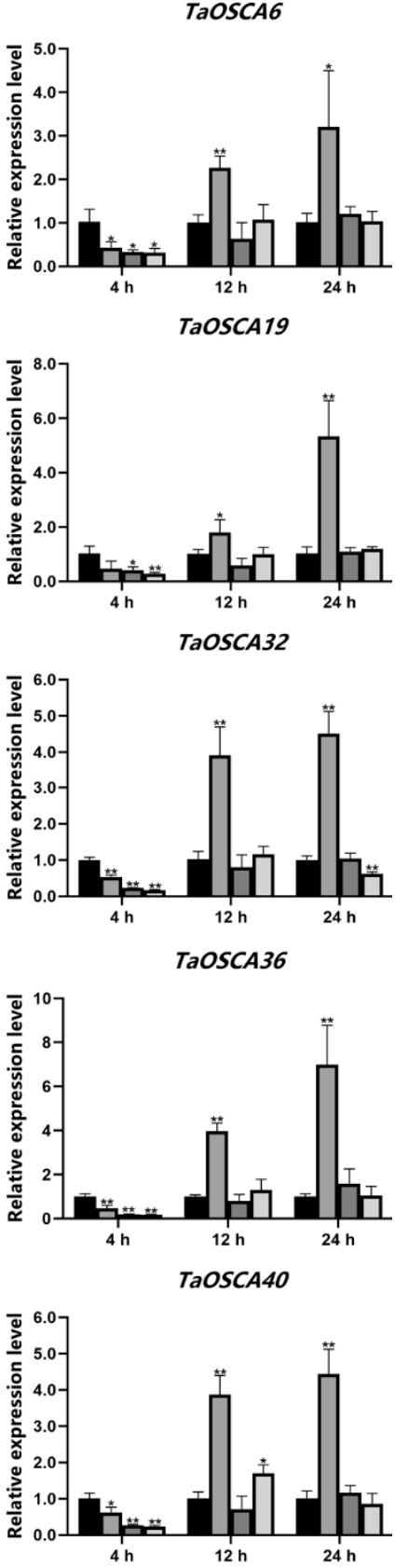

Figure 6. Expression analysis of $\mathrm{TaOSCAs}$ under PEG, $\mathrm{NaCl}$, and ABA treatment. Gene expression was normalized to control unstressed expression level, which was assigned a value of 1 . Data represent average of three independent experiments \pm SD. Standard errors are shown as bars above columns. ${ }^{*}$ denotes significant difference at $p<0.05$, and ${ }^{* *}$ denotes significant difference at $p<0.01$ according to the Student's $t$-test.

\section{Discussion}

Water is essential for the growth and development of plants. The lack of environmental water triggers an osmotic stress signal cascade, which induces short-term cellular responses to reduce water loss, and long-term responses to reshape the transcription network and physiological development processes [31,32]. The perception of biotic and abiotic stresses often leads to the closure of plant stomata $[1,22]$. The rapid influx of $\mathrm{Ca}^{2+}$ through the plasma membrane plays an important role in this reaction $[23,24]$. Current research suggests that OSCA family members act as osmotic sensors to mediate the increase in intracellular 
$\mathrm{Ca}^{2+}$ concentration induced by hyperosmolality and control the closure of stomata in the process of immune signal transduction $[15,25]$. In view of the importance of the functions of OSCA family members, they have been systematically identified in several species, including A. thaliana, rice, maize, and Vigna radiata (L.) [15-18] but not so far in wheat. In this study, 42 OSCA members were identified using T. aestivum genomic data, and the expression patterns of 15 members in different tissues and under three abiotic stress treatments were analyzed. The results of the study lay the foundation for further research on the function of OSCA genes and the cultivation of new T. aestivum varieties.

The number of OSCAs in the T. aestioum genome is more than that of $A$. thaliana, rice, maize, and Vigna radiate [15-18], perhaps in part because the T. aestivum genome is larger. Phylogenetic analysis showed that TaOSCAs fall into four clades (Figure 1), consistent with the topology of the phylogenetic tree of A. thaliana OSCA members [15]. The TaOSCAs in clades I, II, and III are relatively highly conserved, and the composition patterns of the conserved motifs in the three branches are similar (Figure 2A,B). The TaOSCAs in clade IV have only four conserved motifs, much less than other TaOSCAs, and the gene structure is quite different from the members of the other clades (Figure 2). Interestingly, the three members of this clade have one less pfam13967 domain than other members in terms of domain composition (Figure 3), suggesting that these three members may have unique functions or special functional mechanisms.

The cis-acting elements predicted in the TaOSCA promoter region included environmental stress-related elements, hormone-responsive elements, development-related elements, and light-responsive elements (Figure 4A). Environmental stress-related elements include STRE/DRE1/ARE/TC-rich repeats and so on (Figure 4B). STRE elements are activated by heat shock, osmotic stress, low $\mathrm{pH}$, nutrient starvation, etc. DRE1 is induced by drought and osmotic stress, ARE is induced by anaerobiosis, and TC-rich repeats play a role in response to defense [33]. This suggests that TaOSCA genes are involved in the signal transduction network of various stresses in T. aestivum. Consistent with this, the expression of multiple genes was significantly up-regulated under PEG/ABA/NaCl treatment. For example, TaOSCA21/-39 were up-regulated by about 10 times when treated with PEG for $24 \mathrm{~h}$, while these two genes were up-regulated by about five times when treated with ABA for $24 \mathrm{~h}$ (Figure 6), indicating that these two members can respond simultaneously to PEG and ABA.

Studies on the members of the OSCA family in A. thaliana show that different members participate in response to different stress events [15,21,25]. For example, OSCA3.1 has been reported to be an early response protein to dehydration stress [21]. OSCA1.1 is a hyperosmolality sensor, and OSCA1.3 controls the influx of $\mathrm{Ca}^{2+}$ in the process of immune signal transduction [15,25]. A similar differentiation in function was observed in our gene expression results. TaOSCA21/-39 were significantly induced by PEG and ABA but were inhibited by $\mathrm{NaCl}$ treatment for $4 \mathrm{~h}$ (Figure 6). Following $\mathrm{NaCl}$ treatment for $24 \mathrm{~h}$, there was no significant effect on the expression of TaOSCA21, but TaOSCA39 was upregulated by two times (Figure 6). TaOSCA40 had no obvious response to $\mathrm{NaCl}$, but its expression was up-regulated by two times when treated with ABA for $12 \mathrm{~h}$ (Figure 6). The number of development-related elements is less than the other three types of elements (Figure 4A), suggesting that the main function of TaOSCAs is to mediate environmental stress signal transduction.

\section{Materials and Methods}

\subsection{TaOSCA Gene Identification}

The protein sequences of 15 A thaliana OSCA genes were downloaded from TAIR. The newly released reference genome of bread wheat (Triticum aestivum) used in this study was downloaded from the Ensemble Plants database (http://plants.ensembl.org/Triticum aestivum/Info/Index, accessed on 29 December 2021). TaOSCA was identified through two rounds of BLASTP referring to Wu et al. and Chen et al. [34,35]. Firstly, all A. thaliana OSCA sequences were used to search for possible TaOSCA sequences through TBtools [36]. Then, 
NCBI's Batch CD-Search function was used to confirm whether the candidate TaOSCA had the conserved DUF221 domain (pfam accession number: 02714) and other typical domains. Those that did not meet the candidate conditions were eliminated. The predicted CDS length, PI, and molecular weight of the TaOSCA proteins were determined by ExPASy [37].

\subsection{Phylogenetic Analysis}

AtOSCA and TaOSCA full-length protein sequences were used to construct phylogenetic trees in MEGAX using neighbor-joining (NJ) method with 1000 bootstrap replicates [38].

\subsection{Conserved Motifs, Gene Structure, and Functional Domain Analysis}

Conserved motifs were analyzed with the MEME program, and the maximum number of predicted motifs was set to 10 [39]. The gene structure was analyzed using the T. aestivum genome annotation files (downloaded from the Ensemble Plants database) and visualized with TBtools. The NCBI Batch CD-Search function was used to analyze and visualize the functional domains.

\subsection{Promoter Cis-Acting Element Prediction}

In order to study the cis-elements in the promoter region of the TaOSCA gene, the $2 \mathrm{~kb}$ genomic sequence upstream of the start codon (ATG) of each gene was downloaded from the Ensemble Plants database. The putative cis-regulatory elements in the promoter sequence were analyzed by PlantCARE [33].

\subsection{Plant Materials and Abiotic Stress Treatments}

For quantitative PCR expression analysis, T. aestivum seedlings (YangMai 158, shared by Professor Jian Yang from Ningbo University) were raised in potting compost under the following controlled conditions: temperature $23 \pm 1{ }^{\circ} \mathrm{C} ; 16 \mathrm{~h}$ day $/ 8 \mathrm{~h}$ night; and relative humidity $60 \%$. Seedlings at the three-leaf stage were treated with $20 \%$ (w/v) PEG-6000, $\mathrm{NaCl}(100 \mathrm{mM}), \mathrm{ABA}(100 \mu \mathrm{M})$, or $0.1 \%$ Triton $\mathrm{X}-100$ (control) [17,40]. After the stress treatment, leaves were collected at different time points, quickly frozen in liquid nitrogen, and stored at $-80{ }^{\circ} \mathrm{C}$. RNA was then extracted to analyze the expression patterns of different OSCA genes.

\subsection{RNA Isolation and Expression Analysis of TaOSCA Genes}

Total RNA was extracted by the TRIZOL method, and $1 \mu \mathrm{g}$ total RNA was used for reverse transcription using Toyobo cDNA First Strand Synthesis Kit. Subsequently, RT-qPCR was performed on the Roche LightCycler ${ }^{\circledR} 480$ Real-Time PCR instrument with Toyobo Premix Kit. Three independent biological replicates and three technical replicates were adopted. The T. aestivum cell division cycle (CDC) gene (accession number XM_020313450) was used as the internal reference gene, and the relative expression of the gene was calculated by the $2^{-\Delta \Delta C(t)}$ method [41]. All primers are listed in the Supplementary Table S4.

\section{Conclusions}

In this study, we identified 42 OSCA members of T. aestivum and systematically analyzed their phylogeny, gene structure, conserved domains, cis-acting elements, tissuespecific expression, and transcriptional response to different abiotic stresses. The results show that all $15 \mathrm{TaOSCA}$ members tested responded to PEG treatment, while TaOSCA12/-39 responded simultaneously to PEG and ABA, indicating that T. aestivum OSCA genes play important roles in regulating the response of plants to various abiotic stresses. The whole genome identification and characterization of the members of the OSCA family in T. aestivum is an important starting point for further in-depth study of the function of the gene family and lays the foundation for the breeding and genetic improvement of T. aestivum. 
Supplementary Materials: The following supporting information can be downloaded at: https:// www.mdpi.com/article/10.3390/ijms23010469/s1.

Author Contributions: Conceptualization, S.R. and J.C.; methodology, X.W., L.H. and S.Q.; investigation, K.T., S.L. and L.C.; writing—original draft preparation, S.R.; writing-review and editing, J.C.; supervision, J.C.; funding acquisition, J.C. All authors have read and agreed to the published version of the manuscript.

Funding: This work was financially supported by China Agriculture Research System from the Ministry of Agriculture of the P.R. China (CARS-03) and K. C. Wong Magna Fund in Ningbo University. The funders did not participate in the design of this study, data collection, analysis, or manuscript writing.

Institutional Review Board Statement: Not applicable.

Informed Consent Statement: Not applicable.

Data Availability Statement: Not applicable.

Acknowledgments: We thank M. J. Adams, Minehead, UK, for critical reading of the manuscript.

Conflicts of Interest: The authors declare no conflict of interest. The funders had no role in the design of the study; in the collection, analyses, or interpretation of data; in the writing of the manuscript; or in the decision to publish the results.

\section{References}

1. Melotto, M.; Zhang, L.; Oblessuc, P.R.; He, S.Y. Stomatal defense a decade later. Plant Physiol. 2017, 174, 561-571. [CrossRef]

2. Chen, X.; Ding, Y.; Yang, Y.; Song, C.; Wang, B.; Yang, S.; Guo, Y.; Gong, Z. Protein kinases in plant responses to drought, salt, and cold stress. J. Integr. Plant Biol. 2021, 63, 53-78. [CrossRef]

3. Bartels, D.; Sunkar, R. Drought and salt tolerance in plants. CRC Crit. Rev. Plant Sci. 2005, 24, 23-58. [CrossRef]

4. McAinsh, M.R.; Pittman, J.K. Shaping the calcium signature. New Phytol. 2009, 181, 275-294. [CrossRef] [PubMed]

5. Kaur, H.; Sirhindi, G.; Bhardwaj, R.; Alyemeni, M.N.; Siddique, K.H.; Ahmad, P. 28-homobrassinolide regulates antioxidant enzyme activities and gene expression in response to salt and temperature-induced oxidative stress in Brassica juncea. Sci. Rep. 2018, 8, 8735. [CrossRef]

6. Berridge, M.J.; Lipp, P.; Bootman, M.D. The versatility and universality of calcium signalling. Nat. Rev. Mol. Cell Biol. 2000, 1, 11-21. [CrossRef] [PubMed]

7. Knight, H.; Trewavas, A.J.; Knight, M.R. Calcium signalling in Arabidopsis thaliana responding to drought and salinity. Plant J 1997, 12, 1067-1078. [CrossRef]

8. Dodd, A.N.; Kudla, J.; Sanders, D. The language of calcium signaling. Annu. Rev. Plant Biol. 2010, 61, 593-620. [CrossRef] [PubMed]

9. Kung, C. A possible unifying principle for mechanosensation. Nature 2005, 436, 647-654. [CrossRef]

10. Arnadottir, J.; Chalfie, M. Eukaryotic mechanosensitive channels. Annu. Rev. Biophys. 2010, 39, 111-137. [CrossRef]

11. Zhu, J.K. Salt and drought stress signal transduction in plants. Annu. Rev. Plant Biol. 2002, 53, 247-273. [CrossRef] [PubMed]

12. Monshausen, G.B.; Gilroy, S. Feeling green: Mechanosensing in plants. Trends Cell Biol. 2009, 19, 228-235. [CrossRef] [PubMed]

13. Swarbreck, S.M.; Colaco, R.; Davies, J.M. Plant calcium-permeable channels. Plant Physiol. 2013, 163, 514-522. [CrossRef] [PubMed]

14. Hou, C.; Tian, W.; Kleist, T.; He, K.; Garcia, V.; Bai, F.; Hao, Y.; Luan, S.; Li, L. DUF221 proteins are a family of osmosensitive calcium-permeable cation channels conserved across eukaryotes. Cell Res. 2014, 24, 632-635. [CrossRef] [PubMed]

15. Yuan, F.; Yang, H.; Xue, Y.; Kong, D.; Ye, R.; Li, C.; Zhang, J.; Theprungsirikul, L.; Shrift, T.; Krichilsky, B.L.; et al. OSCA1 mediates osmotic-stress-evoked $\mathrm{Ca}^{2+}$ increases vital for osmosensing in Arabidopsis. Nature 2014, 514, 367-371. [CrossRef] [PubMed]

16. Li, Y.; Yuan, F.; Wen, Z.; Li, Y.; Wang, F.; Zhu, T.; Zhuo, W.; Jin, X.; Wang, Y.; Zhao, H.; et al. Genome-wide survey and expression analysis of the OSCA gene family in rice. BMC Plant Biol. 2015, 15, 261. [CrossRef]

17. Yin, L.; Zhang, M.; Wu, R.; Chen, X.; Liu, F.; Xing, B. Genome-wide analysis of OSCA gene family members in Vigna radiata and their involvement in the osmotic response. BMC Plant Biol. 2021, 21, 1-12. [CrossRef]

18. Ding, S.; Feng, X.; Du, H.; Wang, H. Genome-wide analysis of maize OSCA family members and their involvement in drought stress. Peer] 2019, 7, e6765. [CrossRef] [PubMed]

19. Schroeder, B.C.; Cheng, T.; Jan, Y.N.; Jan, L.Y. Expression cloning of TMEM16A as a calcium-activated chloride channel subunit. Cell 2008, 134, 1019-1029. [CrossRef]

20. Liu, X.; Wang, J.; Sun, L. Structure of the hyperosmolality-gated calcium-permeable channel OSCA1.2. Nat. Commun. 2018, 9, 5060. [CrossRef]

21. Kiyosue, T.; Yamaguchi-Shinozaki, K.; Shinozaki, K. Cloning of cDNAs for genes that are early-responsive to dehydration stress (ERDs) in Arabidopsis thaliana L.: Identification of three ERDs as HSP cognate genes. Plant Mol. Biol. 1994, 25, 791-798. [PubMed] 
22. Sussmilch, F.C.; Schultz, J.; Hedrich, R.; Roelfsema, M.R.G. Acquiring control: The evolution of stomatal signalling pathways. Trends Plant. Sci. 2019, 24, 342-351. [CrossRef] [PubMed]

23. Hedrich, R. Ion channels in plants. Physiol. Rev. 2012, 92, 1777-1811. [CrossRef] [PubMed]

24. Jezek, M.; Blatt, M.R. The membrane transport system of the guard cell and its integration for stomatal dynamics. Plant Physiol. 2017, 174, 487-519. [CrossRef]

25. Thor, K.; Jiang, S.; Michard, E.; George, J.; Scherzer, S.; Huang, S.; Dindas, J.; Derbyshire, P.; Leitão, N.; DeFalco, T.A.; et al. The calcium-permeable channel OSCA1.3 regulates plant stomatal immunity. Nature 2020, 585, 569-573. [CrossRef] [PubMed]

26. Pfeifer, M.; Kugler, K.G.; Sandve, S.R.; Zhan, B.; Rudi, H.; Hvidsten, T.R.; Mayer, K.F.X.; Olsen, O.-A.; International Wheat Genome Sequencing Consortium. Genome interplay in the grain transcriptome of hexaploid bread wheat. Science 2014, 345, 1250091. [CrossRef] [PubMed]

27. Wadskog, I.; Forsmark, A.; Rossi, G.; Konopka, C.; Öyen, M.; Goksör, M.; Ronne, H.; Brennwald, P.; Adler, L. The yeast tumor suppressor homologue Sro7p is required for targeting of the sodium pumping ATPase to the cell surface. Mol. Biol. Cell 2006, 17, 4988-5003. [CrossRef]

28. Zhu, J.; Zhang, B.; Smith, E.N.; Drees, B.; Brem, R.B.; Kruglyak, L.; Bumgarner, R.E.; Schadt, E.E. Integrating large-scale functional genomic data to dissect the complexity of yeast regulatory networks. Nat. Genet. 2008, 40, 854-861. [CrossRef]

29. Wang, W.; Vinocur, B.; Altman, A. Plant responses to drought, salinity and extreme temperatures: Towards genetic engineering for stress tolerance. Planta 2003, 218, 1-14. [CrossRef]

30. Chen, K.; Li, G.J.; Bressan, R.A.; Song, C.P.; Zhu, J.K.; Zhao, Y. Abscisic acid dynamics, signaling, and functions in plants. J. Integr. Plant Biol. 2020, 62, 25-54. [CrossRef]

31. Cutler, S.R.; Rodriguez, P.L.; Finkelstein, R.R.; Abrams, S.R. Abscisic acid: Emergence of a core signaling network. Annu. Rev. Plant Biol. 2010, 61, 651-679. [CrossRef]

32. Kim, T.H.; Böhmer, M.; Hu, H.; Nishimura, N.; Schroeder, J.I. Guard cell signal transduction network: Advances in understanding abscisic acid, $\mathrm{CO}_{2}$, and $\mathrm{Ca}^{2+}$ signaling. Annu. Rev. Plant Biol. 2010, 61, 561-591. [CrossRef] [PubMed]

33. Lescot, M.; Déhais, P.; Thijs, G.; Marchal, K.; Moreau, Y.; Van de Peer, Y.; Rouze, P.; Rombauts, S. PlantCARE, a database of plant cis-acting regulatory elements and a portal to tools for in silico analysis of promoter sequences. Nucleic Acids Res. 2002, 30, 325-327. [CrossRef] [PubMed]

34. Wu, X.; Lai, Y.; Lv, L.; Ji, M.; Han, K.; Yan, D.; Lu, Y.; Peng, J.; Rao, S.; Yan, F.; et al. Fasciclin-like arabinogalactan gene family in Nicotiana benthamiana: Genome-wide identification, classification and expression in response to pathogens. BMC Plant Biol. 2020, 20, 305. [CrossRef] [PubMed]

35. Chen, X.; Wu, X.; Qiu, S.; Zheng, H.; Lu, Y.; Peng, J.; Wu, G.; Chen, J.; Rao, S.; Yan, F. Genome-wide identification and expression profiling of the BZR transcription factor gene family in Nicotiana benthamiana. Int. J. Mol. Sci. 2021, 22, 10379. [CrossRef]

36. Chen, C.; Chen, H.; Zhang, Y.; Thomas, H.R.; Frank, M.H.; He, Y.; Xia, R. TBtools: An integrative toolkit developed for interactive analyses of big biological data. Mol. Plant 2020, 13, 1194-1202. [CrossRef]

37. Wilkins, M.R.; Gasteiger, E.; Bairoch, A.; Sanchez, J.-C.; Williams, K.L.; Appel, R.D.; Hochstrasser, D.F. Protein identification and analysis tools in the ExPASy server. Methods Mol. Biol. 1999, 112, 531-552.

38. Kumar, S.; Stecher, G.; Li, M.; Knyaz, C.; Tamura, K. MEGA X: Molecular evolutionary genetics analysis across computing platforms. Mol. Biol. Evol. 2018, 35, 1547-1549. [CrossRef]

39. Bailey, T.L.; Boden, M.; Buske, F.A.; Frith, M.; Grant, C.E.; Clementi, L.; Ren, J.; Li, W.W.; Noble, W.S. MEME Suite: Tools for motif discovery and searching. Nucleic Acids Res. 2009, 37, W202-W208. [CrossRef]

40. He, L.; Chen, X.; Xu, M.; Liu, T.; Zhang, T.; Li, J.; Yang, J.; Chen, J.; Zhong, K. Genome-wide identification and characterization of the cystatin gene family in bread wheat (Triticum aestivum L.). Int. J. Mol. Sci. 2021, 22, 264. [CrossRef]

41. Livak, K.J.; Schmittgen, T.D. Analysis of relative gene expression data using real-time quantitative PCR and the $2^{-\Delta \Delta C T}$ Method. Methods 2001, 25, 402-408. [CrossRef] [PubMed] 